\title{
Effects of Band Nonparabolicity and Band Offset on the Electron Gas Properties in InAs/AISb Quantum Well
}

\author{
Gafur Gulyamov¹, Bahrom Toshmirza 0'g'li Abdulazizov², Baymatov Paziljon Jamoldinovich² \\ ${ }^{1}$ Namangan Engineering-Pedagogical Institute, Namangan, Uzbekistan \\ ${ }^{2}$ Namangan State University, Namangan, Uzbekistan \\ Email: Gulyamov1949@mail.ru
}

How to cite this paper: Gulyamov, G., Abdulazizov, B.T. and Jamoldinovich, B.P. (2016) Effects of Band Nonparabolicity and Band Offset on the Electron Gas Properties in InAs/ AlSb Quantum Well. Journal of Modern Physics, 7, 1644-1650.

http://dx.doi.org/10.4236/jmp.2016.713149

Received: July 13, 2016

Accepted: September 10, 2016

Published: September 13, 2016

Copyright $\odot 2016$ by authors and Scientific Research Publishing Inc. This work is licensed under the Creative

Commons Attribution International License (CC BY 4.0).

http://creativecommons.org/licenses/by/4.0/

\begin{abstract}
One-band effective mass model is used to simulation of electron gas properties in quantum well. We calculate of dispersion curves for first three subbands. Calculation results of Fermi energy, effective mass at Fermi level as function of electron concentration are presented. The obtained results are good agreement with the experimental dates.
\end{abstract}

\section{Keywords}

Quantum Well, In-Plane Dispersion, InAs, AlSb, Two Dimentional Electron Gas, Effective Mass, Cyclotron Mass

\section{Introduction}

In semiconductors, InAs and InSb of the conduction band are characterized by a strong nonparabolicity and recently intensively studied heterostructures based on them [1]-[3]. Nonparabolicity of the conduction band and the nature of the spin splitting of the electron in the quantum well (QW) are studied by the cyclotron resonance [4]-[7].

In [8] has been investigated InAs/AISb based QW with well width $L=15 \mathrm{~nm}$, where two dimensional (2D) electron concentration ranges from $2.7 \times 10^{11}$ to $8 \times 10^{12} \mathrm{~cm}^{-2}$. In this work has been found increase of the effective mass of almost 2 times.

The purpose of this work - the calculation of: 1) subbands dispersion curves, 2) the density of states of 2D electron gas and 3) concentration dependence of effective mass in Fermi level for InAs/AlAs QW with width $L=15 \mathrm{~nm}$.

It is shown that an abrupt change in the density of states leads to a peculiar change in 
the concentration dependence of effective mass.

\section{The In-Plane Dispersion}

Consider a single QW with width $L$ (area $A-\operatorname{In} A s$ ), concluded between barriers with height $V$ (area $B-A I A s)$. The energy is measured from the bottom of the band of the bulk InAs.

In the one band effective mass approximation, the solution of the three-dimensional Schrödinger equation can be represented as $\psi=\mathrm{e}^{i\left(k_{x} x+k_{y} y\right)} \phi(z)$. Then for the area $A$ and $B$, respectively, we can write the following one-dimensional equations

$$
\begin{aligned}
& \frac{\partial^{2} \phi_{A}(z)}{\partial z^{2}}+q^{2} \phi_{A}(z)=0, \quad q=\sqrt{\frac{2 m_{A}(E)}{\hbar^{2}} E-k^{2}} \\
& \frac{\partial^{2} \phi_{B}(z)}{\partial z^{2}}+\chi^{2} \phi_{B}(z)=0, \quad \chi=\sqrt{\frac{2 m_{B}(E)}{\hbar^{2}}(V-E)+k^{2}}
\end{aligned}
$$

Here $k^{2}=k_{x}^{2}+k_{y}^{2}$ and $k-$ is in plane wave vector, $m_{A, B}(E)$-energy-dependent effective mass of the electrons in the material $A$ or $B$. Solving Equations (1) and (2), using the boundary condition

$$
\begin{aligned}
& \phi_{A}(0)=\phi_{B}(0),\left.\frac{1}{m_{A}(E)} \frac{\mathrm{d} \phi_{A}(z)}{\mathrm{d} z}\right|_{z=0}=\left.\frac{1}{m_{B}(E)} \frac{\mathrm{d} \phi_{B}(z)}{\mathrm{d} z}\right|_{z=0} \\
& \phi_{A}(L)=\phi_{B}(L),\left.\frac{1}{m_{A}(E)} \frac{\mathrm{d} \phi_{A}(z)}{\mathrm{d} z}\right|_{z=L}=\left.\frac{1}{m_{B}(E)} \frac{\mathrm{d} \phi_{B}(z)}{\mathrm{d} z}\right|_{z=L}
\end{aligned}
$$

we find the dispersion equation

$$
E=E_{\|}+E_{0}\left[\pi \cdot n-2 \operatorname{Arcsin}\left(\sqrt{\frac{\gamma^{2}\left(E-E_{\|}\right)}{\gamma(\gamma-1) E+\gamma V+\left(1-\gamma^{2}\right) E_{\|}}}\right)\right]^{2}
$$

here $E_{\|}=\hbar^{2} k^{2} / 2 m_{A}(E), \quad E_{0}=\hbar^{2} / 2 m_{A}(E) L^{2}, \gamma=m_{B}(E) / m_{A}(E)$.

Nonparabolicity of conduction band well takes into account by formulas

$$
\begin{aligned}
& \frac{m_{0}}{m_{A}(E)}=1+\frac{E_{P A}}{3}\left[\frac{2}{E+E_{g A}}+\frac{1}{E+E_{g A}+\Delta_{A}}\right] \\
& \frac{m_{0}}{m_{B}(E)}=1+\frac{E_{P B}}{3}\left[\frac{2}{E-V+E_{g B}}+\frac{1}{E-V+E_{g B}+\Delta_{B}}\right]
\end{aligned}
$$

where, $m_{0}$-the free electron mass, $E_{p}$-the Kane parameter, $E_{g}$-the band gap, $\Delta-$ the spin-orbital splitting of valence band, $V$-conduction band offset. Band parameters of InAs and AlSb are shown in Table 1.

To describe the statistics of electrons, Equation (5) is non convenient because it is not solvable with respect to $E$ or $k$. Therefore, we replace Equation (5) is by simple approximation

$$
E \frac{m_{A}(E)}{m_{A}(0)} \approx \frac{\hbar^{2} k^{2}}{2 m_{A}(0)}+E_{n} \frac{m_{A}\left(E_{n}\right)}{m_{A}(0)}
$$


Table 1. Band parameters of InAs and AlSb.

\begin{tabular}{ccc}
\hline & $\operatorname{InAs}(A)$ & $\operatorname{AISb}(B)$ \\
\hline$E_{g}, \mathrm{eV}$ & 0.42 & 2.37 \\
$\Delta, \mathrm{eV}$ & 0.38 & 0.75 \\
$E_{P}, \mathrm{eV}$ & 21.2 & 20.85 \\
$m(0),\left[m_{0}\right]$ & 0.023 & 0.11 \\
$V, \mathrm{eV}$ & 0 & 1.35 \\
\hline
\end{tabular}

where, $E_{n}-$ is bottom of $\mathrm{n}$-th subbands. Now, approximation (8) is the best solution of (5). However, values of $E_{n}$ in (8) now are obtained from Equation (5) at $k=0$ by use numeric method.

For InAs/AISb QW with $L=15 \mathrm{~nm}$, we have: $E_{1}=0.0454 \mathrm{eV}, E_{2}=0.158 \mathrm{eV}, E_{3}=$ $0.304 \mathrm{eV}$, and for case $L=6 \mathrm{~nm}$ we have: $E_{1}=0.163 \mathrm{eV}, E_{2}=0.509 \mathrm{eV}, E_{3}=0.903 \mathrm{eV}$.

Calculated dispersion curves from Equation (5) and approximation (8) are compared in Figure 1(a), Figure 1(b).

From Figure 1(a), Figure 1(b) follows that, the approximation (8) is sufficiently accurate and/or (8) is the best solution of (5) in a wide range of width QW. It is convenient when studying the statistics of electrons, kinetic, optical, or other characteristics of the $2 \mathrm{D}$ electron gas. Inconveniences approximation (8) is such that $E_{n}(n=1,2,3, \cdots)$ depends on $L, V$ and on other parameters of materials $A, B$. Therefore every time when changing these parameters, the value of $E_{n}$ is recalculated from Equation (5) at $k=0$.

\section{The Fermi Energy and Thermodynamic DOS}

The total electron concentration is

$$
n_{s}=N_{0} \sum_{n=1} \int_{E_{n}}^{E_{F}} \mathrm{~d}\left(\frac{\hbar^{2} k^{2}}{2 m_{A}(0)}\right)
$$

where

$$
N_{0}=\frac{m_{A}(0)}{\pi \hbar^{2}}=\frac{m_{A}(0) \cdot 0.413 \times 10^{15}}{\ni \mathrm{B} \cdot \mathrm{cm}^{2}} .
$$

According (8) we have

$$
n_{S}=\sum_{n=1} n_{S}^{(n)}, n_{s}^{(n)}=N_{0}\left[E_{F} \frac{m_{A}\left(E_{F}\right)}{m_{A}(0)}-E_{n} \frac{m_{A}\left(E_{n}\right)}{m_{A}(0)}\right] \theta\left(E_{F}-E_{n}\right)
$$

where $n_{s}^{(n)}$-is $n$-th subband concentration.

In Equation (10), the terms in the sum should be positive. The negative terms in $n_{s}^{(n)}$ excluded by the Heaviside function. It establishes a link between the Fermi energy $E_{F}$ and full 2D electron concentration $n_{s}$. They also determine the concentration of electrons in separate subbands $n_{s}^{(n)}$ for a given $n_{s}$.

From (10), we can estimate the critical concentrations of $n_{c 1}$, in which the Fermi level comes to bottom of the second subband $E_{F}=E_{2}$. In the structure of InAs/AlSb QW 

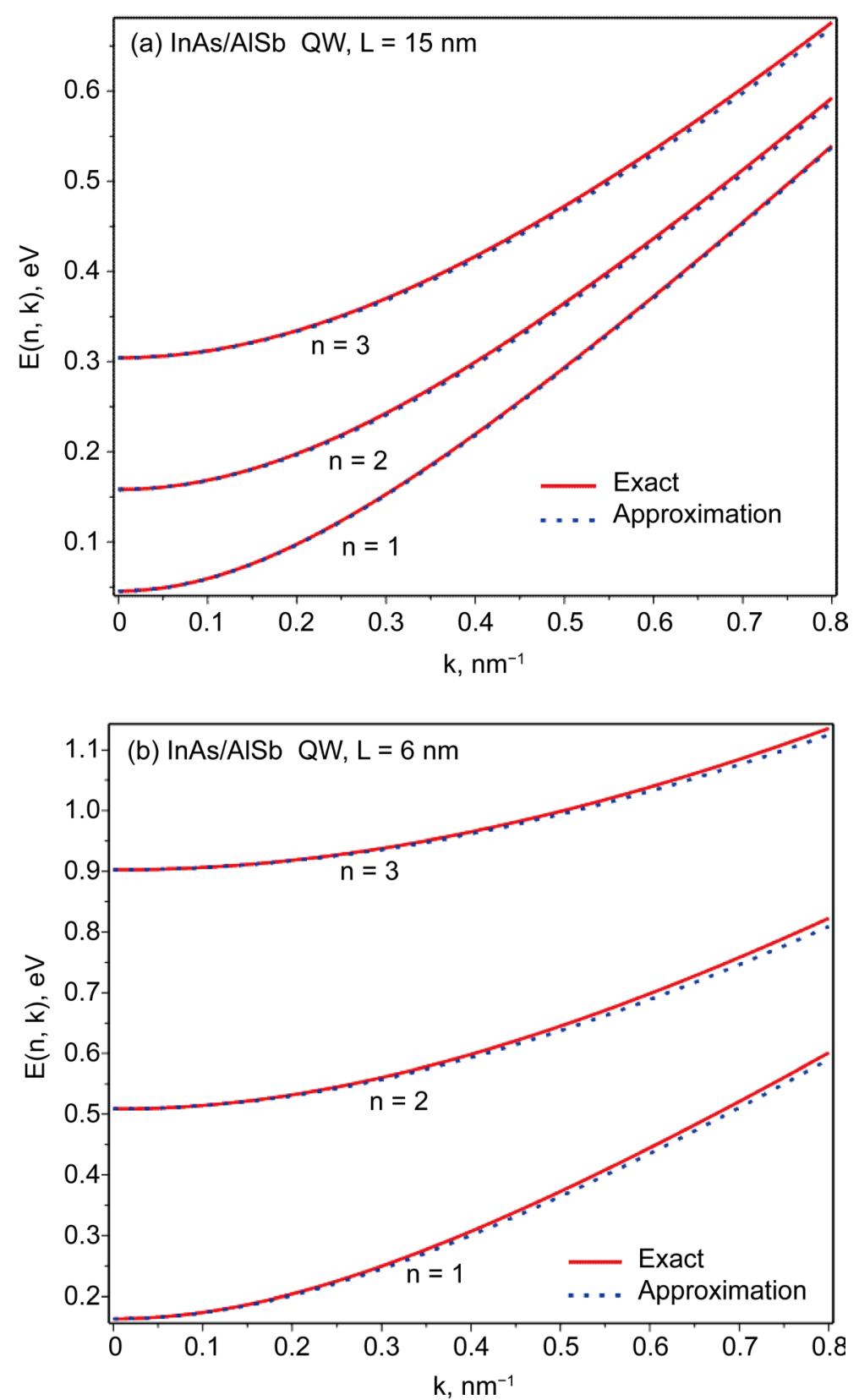

Figure 1. The dispersion curves of the first three subbands $(n=1,2,3)$ in InAs/AISb QW: the full (red) line-according to Equation (5), the dotted (blue) line-approximation (8); (a) $L=15 \mathrm{~nm}$, (b) $L=6 \mathrm{~nm}$.

with the well width $L=15 \mathrm{~nm}$ can be found

$$
n_{c 1}=N_{0}\left[E_{2} \frac{m_{A}\left(E_{2}\right)}{m_{A}(0)}-E_{1} \frac{m_{A}\left(E_{1}\right)}{m_{A}(0)}\right]=1.52 \times 10^{12} \mathrm{~cm}^{-2}
$$

This estimation is close to experimental measured date $n_{c 1, \exp }=1.2 \times 10^{12} \mathrm{~cm}^{-2} \quad[8]$. Similarly, the critical concentration of $n_{c 2}$, in which the Fermi level comes to bottom of the third subband $E_{F}=E_{3}$, is $n_{c 2}=6.87 \times 10^{12} \mathrm{~cm}^{-2}$. 
The dependence $E_{F}\left(n_{s}\right)$ is shown in Figure 2. It is obtained from Equations (10) by changing the Fermi energy in the range of $E_{F}=0 \div 0.33 \mathrm{eV}$. The graph shows that, depending on $E_{F}\left(n_{s}\right)$ there exist a fractures-slowing of increase the Fermi's energy. They are caused by abrupt changes (by jumps), the density of states at the critical points: $n_{s}=n_{c 1}, E_{F}=E_{2}, n_{s}=n_{c 2}, E_{F}=E_{3}$.

These fractures occur at the critical concentrations of $n_{c 1}, n_{c 2} \cdots$, where $E_{F}$ intersects the Fermi level of the bottom of the next subband.

The thermodynamically DOS of electron gas at Fermi level $N_{T}\left(E_{F}\right)=\mathrm{d} n_{S} / \mathrm{d} E_{F}$ is shown in Figure 3.

\section{The Cyclotron Mass}

According approximation (8), the electron effective mass at the Fermi level (cyclotron mass) $m_{c}=\left.\hbar^{2} k(\mathrm{~d} E / \mathrm{d} k)^{-1}\right|_{E=E_{F}}$ is

$$
m_{c}=\left(m_{A}(E)+E \frac{\mathrm{d} m_{A}(E)}{\mathrm{d} E}\right)_{E=E_{F}}
$$

The dependence $m_{c}\left(n_{s}\right)$ is shown in Figure 4.

This dependence can be obtained from Equations (10) and (12) by changing the Fermi energy in the range $E_{F}=0-0.33 \mathrm{eV}$.

This figure shows also the dependence of experimentally measured value of the effective masses (cyclotron mass) $m_{c}$ at the Fermi level of the total concentration of $2 \mathrm{D} n_{s}$ [8]. Fracture in the $m_{c}\left(n_{s}\right)$ occur at critical points: $n_{s}=n_{c 1}, E_{F}=E_{2}$ and $n_{s}=n_{c 2}$, $E_{F}=E_{3}$.

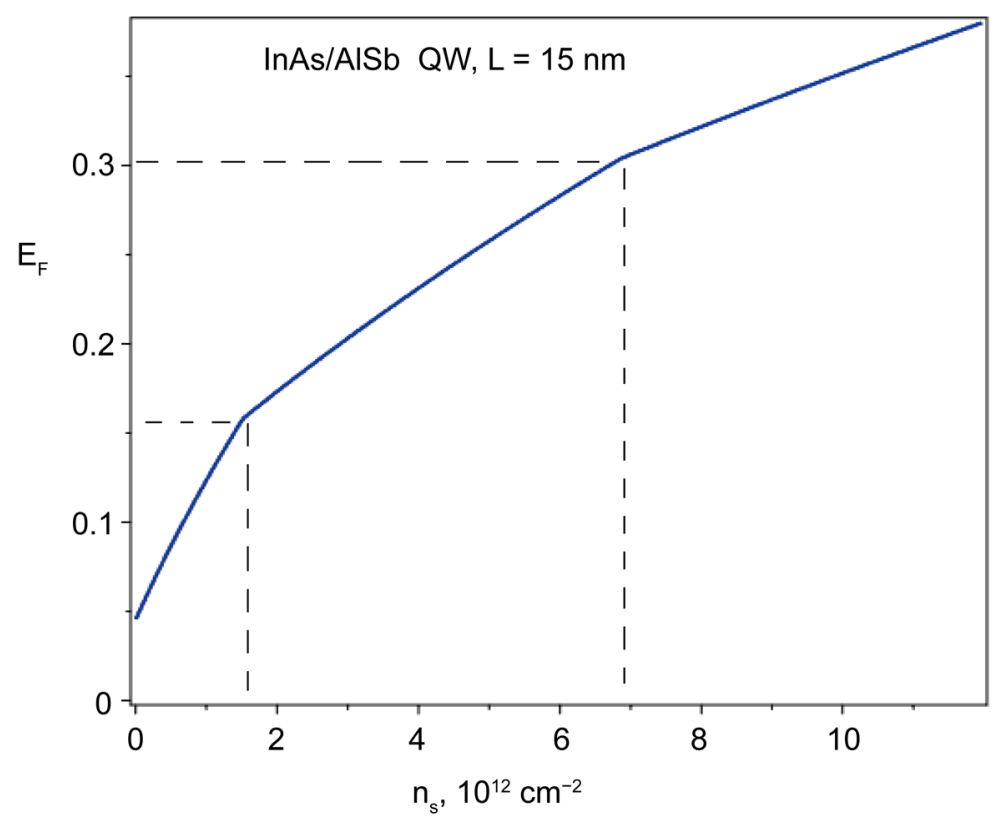

Figure 2. The dependence of the Fermi energy on the $2 \mathrm{D}$ concentration in $\operatorname{In} A s / A I S b \mathrm{QW}$ with $L=$ $15 \mathrm{~nm}, V=1.35 \mathrm{eV}$. The critical points $n_{s}=n_{c 1}, E_{F}=E_{2}$ and $n_{s}=n_{c 2}, E_{F}=E_{3}$ shown by the shaded lines. 


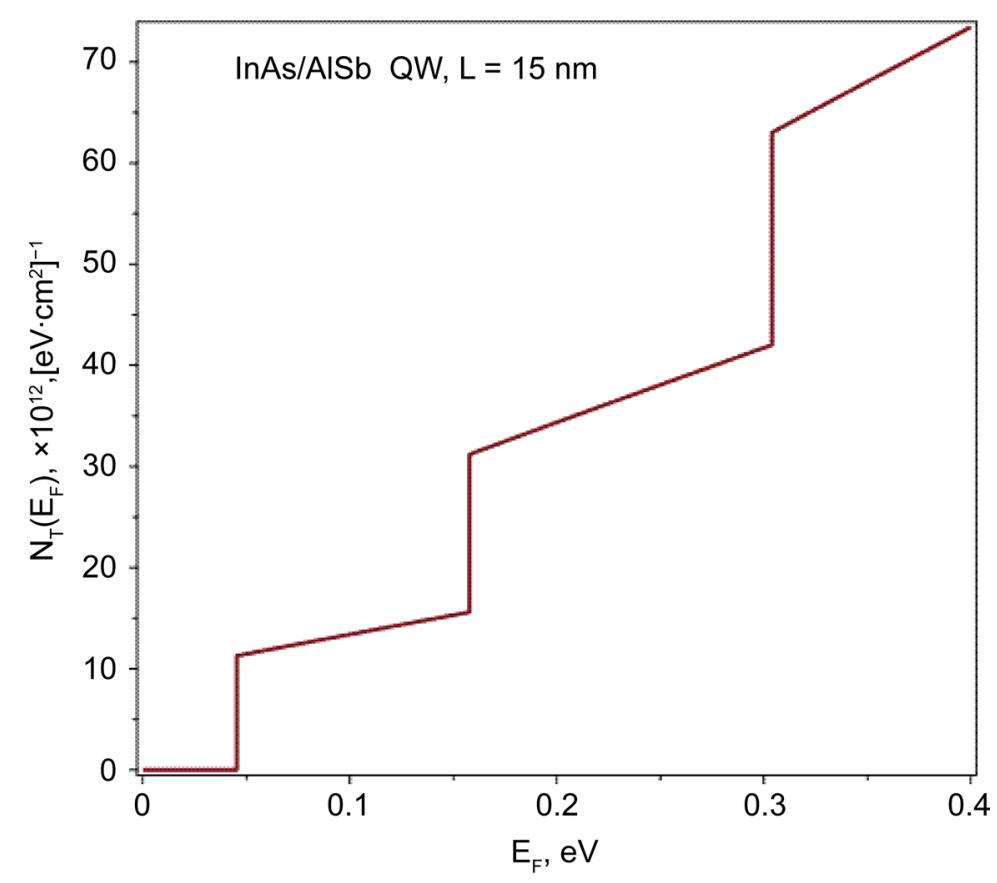

Figure 3. The thermodynamically DOS of electron gas at Fermi level in InAs/AISb QW, $L=15$ $\mathrm{nm}, V=1.35 \mathrm{eV}$.

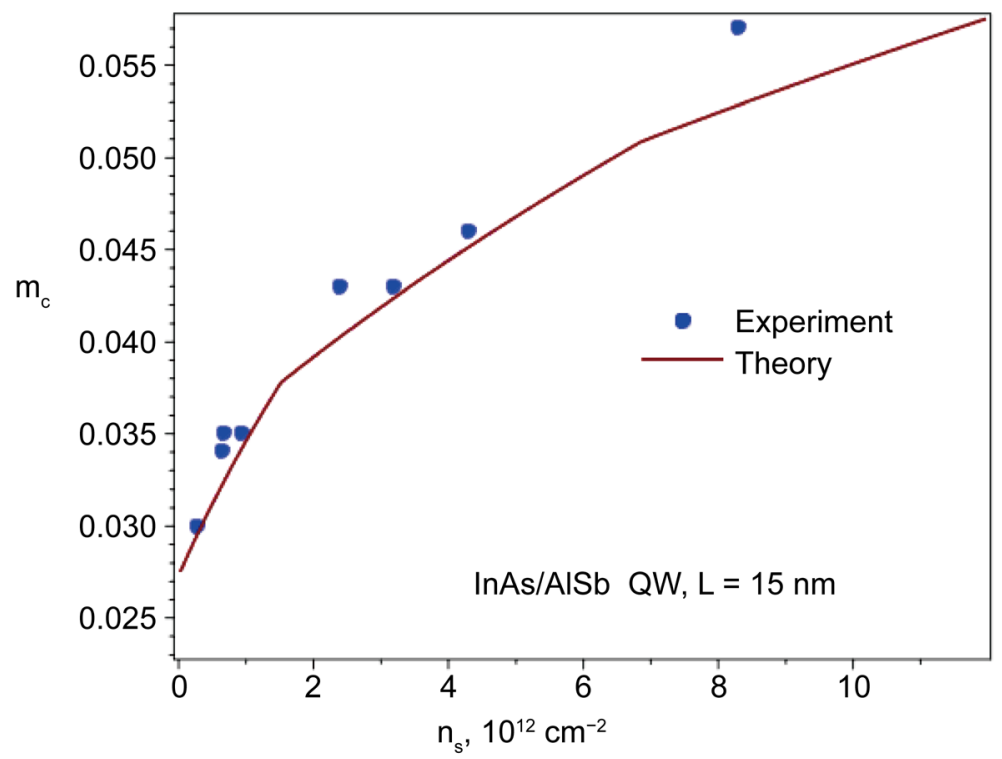

Figure 4. The dependence of the effective mass on the total concentration for InAs/AlSb QW, $L=$ 15 нм, $V=1.35 \mathrm{eV}$.

\section{Conclusion}

In this study are provided useful approximation (8) of subband dispersions and simplified Equation (10) to calculate the statistics of a degenerate electron gas in heterostructured InAs/AlSb QW, which satisfactorily describes the experimental results [8]. They are also useful to study of calculation of the transport, optical and magnetic properties 
of electron gas in a Kane type 2D system. The above description of the algorithms can be applied to other QW heterostructures based on semiconductor group $A_{3} B_{5}$.

\section{Acknowledgements}

This work was supported by the Scientific and Technical program Republic of of Uzbekistan (Grant F2-OT-O-15494).

\section{References}

[1] Vasilyev, Yu.B., Gouider, F., Nachtwei, G. and Buckle, P.D. (2010) Semiconductors, 44, 1511-1514. http://dx.doi.org/10.1134/S1063782610110266

[2] Spirin, K.E., Kalinin, K.P., Krishtopenko, S.S., Maremyanin, K.V., Gavrilenko, V.I. and Sadofyev, Yu.G. (2012) Semiconductors, 46, 1396-1401.

http://dx.doi.org/10.1134/S1063782612110206

[3] Barate, D., Teissier, R., Wang, Y. and Baranov, A.N. (2005) Applied Physics Letters, 87, 051103. http://dx.doi.org/10.1063/1.2007854

[4] Yang, M.J., Lin-Chung, P.J., Wagner, R.J., Waterman, J.R., Moore, W.J. and Shanabrook, B.V. (1993) Semiconductor Science and Technology, 8, S129. http://dx.doi.org/10.1088/0268-1242/8/1S/029

[5] Yang, M.J., Lin-Chung, P.J., Shanabrook, B.V., Waterman, J.R., Wagner, R.J. and Moore, W.J. (1993) Physical Review B, 47, 1691. http://dx.doi.org/10.1103/PhysRevB.47.1691

[6] Gauer, C., Scriba, J., Wixforth, A., Kotthaus, J.P., Bolognesi, C.R., Nguyen, C., Brar, B. and Kroemer, H. (1994) Semiconductor Science and Technology, 9, 1580. http://dx.doi.org/10.1088/0268-1242/9/9/002

[7] Warburton, R.J., Gauer, C., Wixforth, A., Kotthaus, J.P., Brar, B. and Kroemer, H. (1996) Physical Review B, 53, 7903. http://dx.doi.org/10.1103/PhysRevB.53.7903

[8] Aleshkin, V.Ya., Gavrilenko, V.I., Ikonnikov, A.V., Sadofyev, Yu.G., Bird, J.P., Jonhson, S.R. and Zhang, Y.-H. (2005) Semiconductors, 39, 62-66. http://dx.doi.org/10.1134/1.1852647

Submit or recommend next manuscript to SCIRP and we will provide best service for you:

Accepting pre-submission inquiries through Email, Facebook, LinkedIn, Twitter, etc.

A wide selection of journals (inclusive of 9 subjects, more than 200 journals)

Providing 24-hour high-quality service

User-friendly online submission system

Fair and swift peer-review system

Efficient typesetting and proofreading procedure

Display of the result of downloads and visits, as well as the number of cited articles

Maximum dissemination of your research work

Submit your manuscript at: http://papersubmission.scirp.org/ 